OPEN ACCESS

Edited by:

lan Orchard,

University of Toronto, Canada

Reviewed by:

Meet Zandawala,

Brown University, United States

Jean-Paul V. Paluzzi,

York University, Canada

*Correspondence:

Andrew B. Nuss

nuss@cabnr.unr.edu

Monika Gulia-Nuss

mgulianuss@unr.edu

Specialty section

This article was submitted to Neuroendocrine Science,

a section of the journal

Frontiers in Endocrinology

Received: 05 December 2018

Accepted: 28 February 2019

Published: 22 March 2019

Citation:

Sharma $A$, Nuss $A B$ and Gulia-Nuss M (2019) Insulin-Like

Peptide Signaling in Mosquitoes: The

Road Behind and the Road Ahead.

Front. Endocrinol. 10:166.

doi: 10.3389/fendo.2019.00166

\section{Insulin-Like Peptide Signaling in Mosquitoes: The Road Behind and the Road Ahead}

\author{
Arvind Sharma ${ }^{1}$, Andrew B. Nuss ${ }^{1,2 *}$ and Monika Gulia-Nuss ${ }^{1 *}$ \\ ${ }^{1}$ Department of Biochemistry and Molecular Biology, University of Nevada, Reno, NV, United States, ${ }^{2}$ Department of \\ Agriculture, Veterinary, and Rangeland Sciences, University of Nevada, Reno, NV, United States
}

Insulin signaling is a conserved pathway in all metazoans. This pathway contributed toward primordial metazoans responding to a greater diversity of environmental signals by modulating nutritional storage, reproduction, and longevity. Most of our knowledge of insulin signaling in insects comes from the vinegar fly, Drosophila melanogaster, where it has been extensively studied and shown to control several physiological processes. Mosquitoes are the most important vectors of human disease in the world and their control constitutes a significant area of research. Recent studies have shown the importance of insulin signaling in multiple physiological processes such as reproduction, innate immunity, lifespan, and vectorial capacity in mosquitoes. Although insulin-like peptides have been identified and functionally characterized from many mosquito species, a comprehensive review of this pathway in mosquitoes is needed. To fill this gap, our review provides up-to-date knowledge of this subfield.

Keywords: insulin signaling, insulin-like peptides, mosquitoes, insulin receptor, aedes, anopheles, culex

\section{INTRODUCTION}

Insulin-like peptides (ILPs) are broadly conserved among metazoans and are the most studied peptide hormones because of their important regulatory roles in metabolism, growth, and development. All ILPs are 6-8 kDa, share a common structural motif called the insulin fold, and are processed from precursors with similar domain structure (Pre, B, C, A) (1). Among arthropods, insulin signaling is most well-understood in the model insect Drosophila melanogaster. The $D$. melanogaster genome has eight ILPs (dILPs), each with specific tissue expression. For instance, some dILPs originate from the brain and ventral nerve cord, while others are expressed in the midgut, fat body, or imaginal discs (2-4).

Mosquitoes have varying numbers of ILPs (Table 1) ranging from five to eight, and, similar to the situation in D. melanogaster, expression has been detected in the nervous system, fat body, midgut, ovaries, and other tissues. Each mosquito species has a distinct set of ILPs that are of neural origin, while others are expressed in multiple tissues (5, 7-9).

Similar to other metazoans, the mosquito insulin receptor (MIR) is a transmembrane receptor tyrosine kinase (RTK) and consists of a dimer of $\alpha$ and $\beta$-monomers. The $\alpha$-subunits define ILP ligand binding specificity, whereas the $\beta$-subunits mediate the downstream signal to cellular components. The MIR uses insulin receptor substrate (IRS) as an adaptor molecule to initiate signaling (10). Upon binding of the ligand to its receptor, the $\beta$-subunits undergo auto-phosphorylation at specific tyrosine residues. The activated RTK subsequently phosphorylates specific tyrosine residues of the IRS (11). IRS then recruits 
TABLE 1 | Number of insulin-like peptides identified in different mosquito genera and species.

\begin{tabular}{llc}
\hline Mosquito species & Number of ILPs & References \\
\hline Aedes aegypti & Eight: Aa ILP1-8 & $(5)$ \\
Anopheles gambiae & Five: AglLP1/7, 2, 3/6, 4, 5 & $(6)$ \\
Anopheles stephensi & Five: AslLP1-5 & $(7)$ \\
Culex pipiens & Three: (more likely) & (8) \\
\hline
\end{tabular}

downstream factors to the receptor-IRS complex. The phosphorylated tyrosine residues of the receptor-IRS complex interact with phosphatidylinositol-3-kinase (PI3K) proteins $(12,13)$. Recruitment of PI3K results in the formation of the IRS-PI3K complex. Subsequently, PI3K catalyzes synthesis of phosphatidylinositol-3,4,5-trisphosphate (PIP3) from phosphatidylinositol-4,5-bisphosphate (PIP2).

Phosphatase and Tensin homolog (PTEN) is a negative regulator and can reverse this conversion from PIP3 to PIP2 and decrease the level of PIP3 in the cell. The phosphoinositidedependent protein kinase (PDK) responds to the high PIP3 levels by recruiting Akt $(13,14)$. Akt is considered the master regulator kinase because the phosphorylation of Akt affects a number of downstream protein substrates including the target of rapamycin (TOR) $(15,16)$. TOR activation occurs both as a direct downstream event of insulin signaling activation or, independent of Akt, by the availability of amino acids.

TOR and ILP signaling pathways are considered nutritional sensors at the cellular and systemic level, respectively. Aktmediated phosphorylation of forkhead-related FOXO proteins prevents the FOXO transcription factor from being translocated to the nucleus (17-20). FOXO proteins are indispensable in an organism's response to starvation since they promote conservation of energy or even catabolism (21). There has been some work exploring TOR and FOXO signaling in mosquitoes (22-28), but a detailed review is outside the scope of this article.

\section{IDENTIFICATION AND STRUCTURE OF ILPS}

Prior to the identification of mosquito ILPs and MIR, it was well-known that shortly after blood feeding, neurohormones are released from the brain neurosecretory system that stimulate the ovaries to secrete ecdysteroids, which are necessary for vitellogenesis by the fat body. The silkworm Bombyx mori ILP, bombyxin, was demonstrated to stimulate ecdysteroidogenesis in prothoracic glands in silkworm larvae. This led to hypothesis that insulins are involved in regulation of the ecdysteroid pathway in mosquitoes and commercially available porcine and bovine insulin were tested on unfed mosquito ovaries to test this hypothesis. This lead to the discovery of the MIR in Ae. aegypti (29) and the discovery of other components of the insulin signaling pathway followed shortly (30). However, ILP identification in Ae. aegypti lagged by over half a decade (5). The publication of the Anopheles gambiae genome was seminal in the identification of ILPs in mosquitoes. Seven ILP genes corresponding to five unique ILPs (AgILP1-5) and one MIR were identified in the A. gambiae genome (9). Two AgILP genes encode identical B and A peptides therefore seven ILP genes produce five peptides. Genes encoding eight unique ILPs were found in the Ae. aegypti genome (5). Except for AaILP6, seven other Ae. aegypti ILPs had a propeptide structure consistent with that of other invertebrate ILPs. AaILP6 is unique because it has a short $\mathrm{C}$ peptide and an extended A peptide, similar to the vertebrate insulin growth factors (IGFs), however, the $\mathrm{C}$ peptide of AaILP6 had multiple dibasic proteolytic cleavage sites, in contrast to only one in vertebrate IGFs (5). To date, there is no empirical evidence to confirm whether or not the predicted dibasic sites are actually cleaved/processed in Ae. aegypti.

The proximity of AaILP1, 3, and 8 in the genome scaffold suggested that they may form a polycistronic transcription unit controlled by a single promoter. All three of these have independent putative polyadenylation sites and are capped to generate monocistronic mature mRNAs (5). AgILP1/7 and 3/6, the duplicated gene pairs in A. gambiae, and AaILP1 and 3 appear to be orthologs. Phylogenetic analysis supported an evolutionary relationship between AaILP1 and AgILP1/7, as well as between AaILP3 and AgILP3/6. A functional relationship between Anopheles stephensi, Ae. aegypti, and Cx. quinquefasciatus ILP3 was also demonstrated (31). The third member of the Ae. aegypti ILP operon, AaILP8, was not related in sequence to any of the other dipteran ILPs (5).

Sequences encoding full-length transcripts of five ILPs from the A. stephensi genome (AsILP 1-5) and three from Culex pipiens (CpILP1, CpILP2, and CpILP5) were identified $(7,8)$. ILP4 of A. stephensi was highly similar to A. gambiae ILP4 but this ILP does not have an apparent ortholog in either Ae. aegypti or C. pipiens. Ae. aegypti ILP5 and A. gambiae ILP5 share up to $81 \%$ sequence similarity, uncommon for ILPs, and, together with $C x$. pipiens and A. stephensi ILP5, share the unique feature of an additional amino acid between the second and third cysteine residues in the A chain $(5,7)$. DmILP7, an ortholog of AaILP5, also shares this feature and is well-conserved with the mosquito sequences [(5); Figure 1]. AaILP2 and AgILP2 form another related ILP subgroup (Figure 1).

The putative IGF-like ILP, AaILP6, was closely related to another ILP identified as a gene transcript in Aedes subalbatus (5). The remaining two AaILPs, AaILP4 and 7, do not appear to have any dipteran orthologs (Figure 1). This is not surprising considering that both possess an additional amino acid between the third and fourth cysteine residues in the A peptide, a feature not known for other members of the insulin superfamily (5).

A unifying feature of all ILPs is the presence of six conserved cysteine residues that form disulfide bonds between the $\mathrm{B}$ and A chains (Figure 1). However, outside of these core residues, amino acid sequence similarity diverges between the different types of ILPs. Some functional forms of ILPs are clearly conserved throughout the mosquito (such as ILP2, ILP3) and even dipteran lineages (such as mosquito ILP5/DILP7), and form distinct groupings when subjected to neighbor-joining analysis (Figure 2), but the evolutionary relatedness of different ILP isoforms to one another has poor branch support and remains 


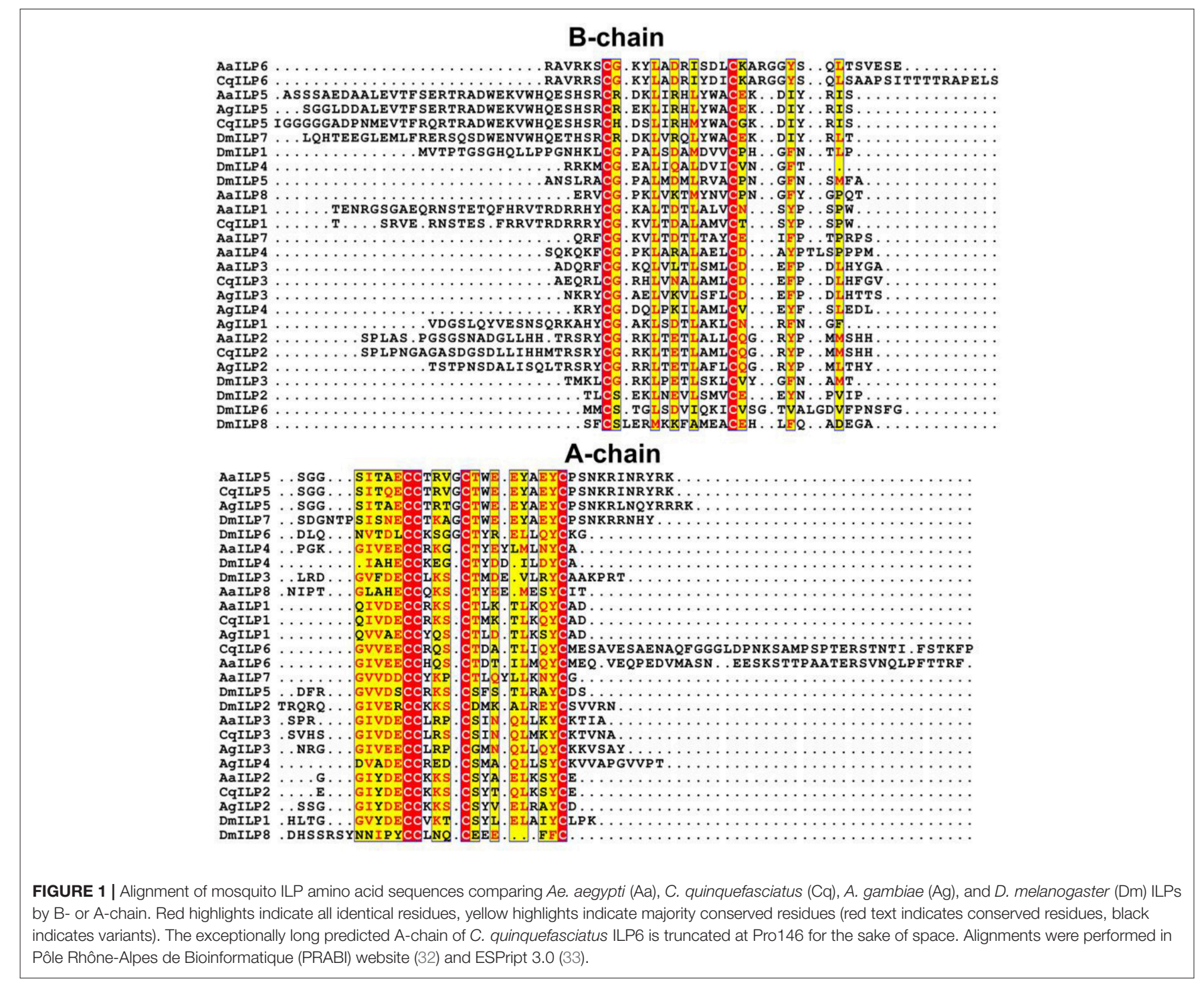

unclear. It is likely that the secondary structure imposed by disulfide bonds and as yet undetermined key functional residues are the most critical components for ILP interaction with the MIR, whereas other amino acids may be more important in preserving spacing in the molecule, rather than the identity of their functional group. This limits our ability to predict functions of ILPs in related species based on amino acid sequence alone.

\section{FUNCTIONS OF INSULIN-LIKE PEPTIDES IN MOSQUITOES}

Function and signaling of ILPs are best characterized for Ae. aegypti [for other reviews see (35-37)]. Unlike D. melanogaster, genetic manipulations of ILPs to study pluripotency in mosquitoes is still in its infancy. The only native ILP isolated so far from mosquitoes is from A. stephensi, AsILP3 (31). AaILP3, AaILP4, and AaILP8 were chemically synthesized $(23,38,39)$ and used to deduce their functions. With the availability of new
CRISPR-Cas9 based gene editing tools, the functions of two additional Ae. aegypti ILPs, AaILP7, and AaILP8, were recently investigated (40) (Table 2, Figure 3).

\section{Nutrient Metabolism}

Among mosquitoes, the role of ILPs in nutrient allocation is best studied in Ae. aegypti. AaILP1, ILP3, and ILP8 are specifically expressed in the brains of adult females (5). Synthetic AaILP3 (sAaILP3) binds to the MIR with high affinity and has been shown as a critical regulator of egg production (23, 38, 39, 41). sAaILP4 and sAaILP8 did not show any competition with sAaILP3 for binding to the MIR (39). Similarly, sAaILP3 (but not sAaILP4 and sAaILP8) injected into mosquitoes decapitated after a sugar meal dose-dependently increased the levels of stored glycogen and lipids and decreased the levels of trehalose (38) in the whole body of females, suggesting its function is analogous to mammalian insulin in vertebrates. 


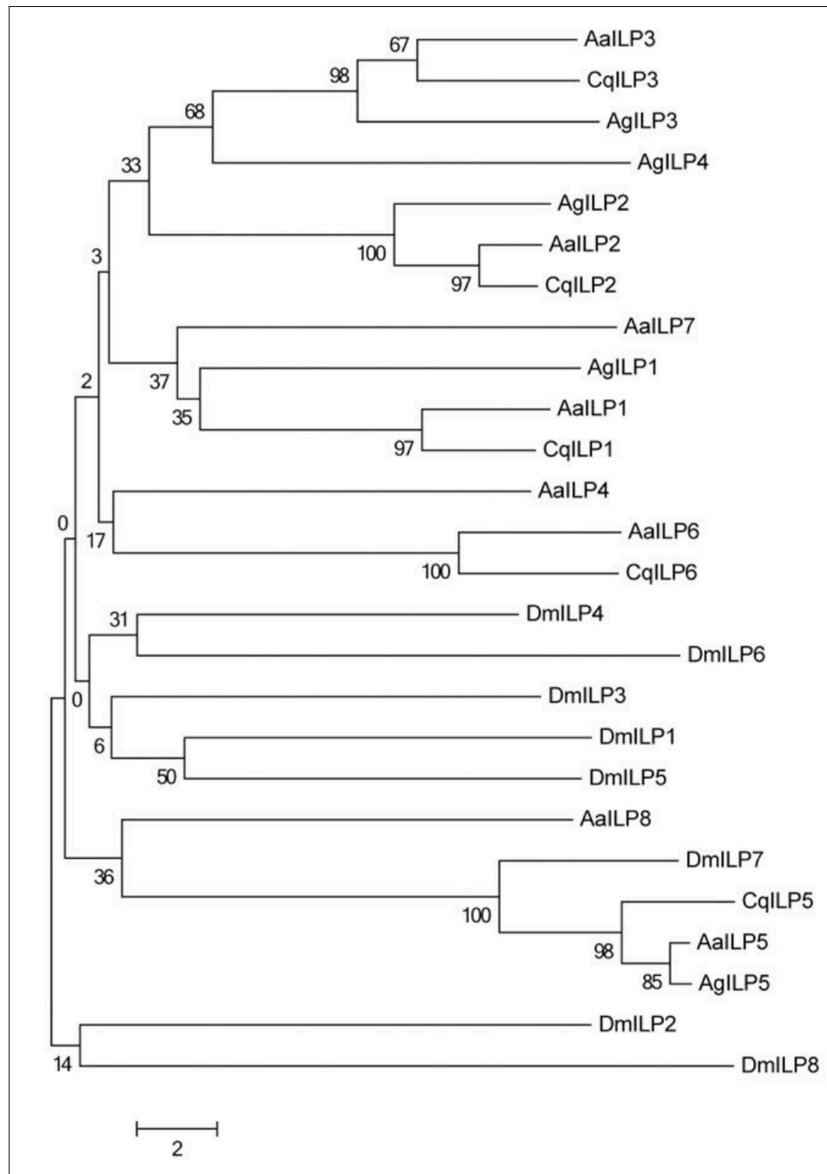

FIGURE 2 | Neighbor-joining tree of mosquito ILP A and B chain amino acid sequences comparing Ae. aegypti (Aa), C. quinquefasciatus (Cq), A. gambiae $(\mathrm{Ag})$, and $D$. melanogaster $(\mathrm{Dm})$. Tree was constructed using MEGA version 6 (34).

AaILP3 transcript levels were higher in mosquitoes that emerged from the high carbohydrate larval diet (43), further suggesting a role in nutrient metabolism. AaILP3 also stimulated the midgut to express trypsin-like proteases that digested the blood meal while amino acid sensing through the target of rapamycin (TOR) pathway enhanced AaILP3, ovary ecdysteroidogenic hormone $(\mathrm{OEH})$, ecdysteroids, and vitellogenin synthesis $(23,47)$.

Some evidence exists that microRNAs regulate AaILP expression. For instance, in the absence of miR277, transcript levels of both AaILP7 and AaILP8 increased in head whereas AaILP1 and AaILP3 transcript levels did not change, suggesting that miR277 targets the first member (AaILP8) of the ILP8ILP1-ILP3 operon (40). CRISPR-Cas9 depletion of AaILP7 and AaILP8 led to metabolic and reproductive defects. A dramatic lipid increase in the fat body in AaILP7 knockouts and a decrease inAaILP8 knockouts suggests a role of these ILPs in modulating lipid deposition and mobilization (40). Glycogen levels exhibited the opposite trends in these mosquitoes, which suggest that both AaILP7 and AaILP8 are involved in lipid and glycogen balance. In
TABLE 2 | Potential functions of mosquito insulin-like peptides.

\begin{tabular}{|c|c|c|}
\hline $\begin{array}{l}\text { Insulin-like } \\
\text { peptide (ILP) }\end{array}$ & Potential function & References \\
\hline \multicolumn{3}{|l|}{ Aedes aegypti } \\
\hline AalLP1 & Not yet studied & \\
\hline AalLP2 & Not yet studied & \\
\hline AalLP3 & $\begin{array}{l}\text { Nutrient metabolism, regulation of } \\
\text { digestive enzymes, Ecdysteroid } \\
\text { production from ovaries, immune response }\end{array}$ & $(23,38,41,42)$ \\
\hline AalLP4 & Nutrient metabolism in males & $(43)$ \\
\hline AalLP5 & Not yet studied & \\
\hline AalLP6 & Not yet studied & \\
\hline AalLP7 & $\begin{array}{l}\text { Glycogen metabolism post blood meal, } \\
\text { nutrient metabolism post blood meal }\end{array}$ & $(40)$ \\
\hline AalLP8 & Hemolymph lipid metabolism, larval molt & $(40,43)$ \\
\hline \multicolumn{3}{|c|}{ Anopheles stephensi } \\
\hline AsILP1 & Not yet studied & \\
\hline AsILP2 & Not yet studied & \\
\hline AsILP3 & Ecdysteroids production by ovaries & $(31)$ \\
\hline AsILP4 & $\begin{array}{l}\text { Ecdysteroids production by ovaries; } \\
\text { Plasmodium falciparum early infection }\end{array}$ & $(31,44)$ \\
\hline AslLP5 & P. falciparum oocyst development & $(44)$ \\
\hline \multicolumn{3}{|c|}{ Anopheles gambiae } \\
\hline AglLP1/7 & Not yet studied & \\
\hline AglLP2 & Not yet studied & \\
\hline AglLP3/6 & P. falciparum infection & $(45)$ \\
\hline AglLP4 & Blood meal nutrients metabolism & $(46)$ \\
\hline AglLP5 & Blood meal nutrients metabolism & $(46)$ \\
\hline \multicolumn{3}{|l|}{ Culex pipiens } \\
\hline CplLP1 & Diapause/overwintering & (8) \\
\hline CplLP2 & Not yet studied & \\
\hline CplLP5 & $\begin{array}{l}\text { Higher expression but not associated with } \\
\text { diapause }\end{array}$ & (8) \\
\hline
\end{tabular}

our study, AaILP8 transcript levels were higher in the late fourth instar larvae suggesting a possible role in larval to pupal molt (43), a function similar to D. melanogaster ILP8 (2).

In A. gambiae, artificial blood meal (albumin and amino acid mixture) rapidly triggered transcription of two ILPs- AgILP3 and AgILP4, in the brain of starved mosquitoes, and the response was higher compared to sucrose fed mosquitoes (46). In A. stephensi, expression of ILPs did not change significantly with age or upon ingestion of a sugar or blood meal $(7,44)$ suggesting differences in mosquito species. ILP functions in nutrient allocation in Culex spp. have not been studied yet.

Insulin receptor knockdown by RNA interference (RNAi) in newly eclosed females and subsequent decapitation within $2 \mathrm{~h}$ post blood meal resulted in slow blood digestion in Ae. aegypti (23). In mosquitoes decapitated post blood meal, sAaILP3 was able to restore trypsin transcripts and enzyme levels, while sAaILP4 and sAaILP8 had no effect on trypsin expression (23). A similar effect of insulin receptor knockdown on midgut trypsin levels was observed in C. quinquefasciatus (48). Whether this phenomenon extends to Anopheles spp. remains to be explored. 


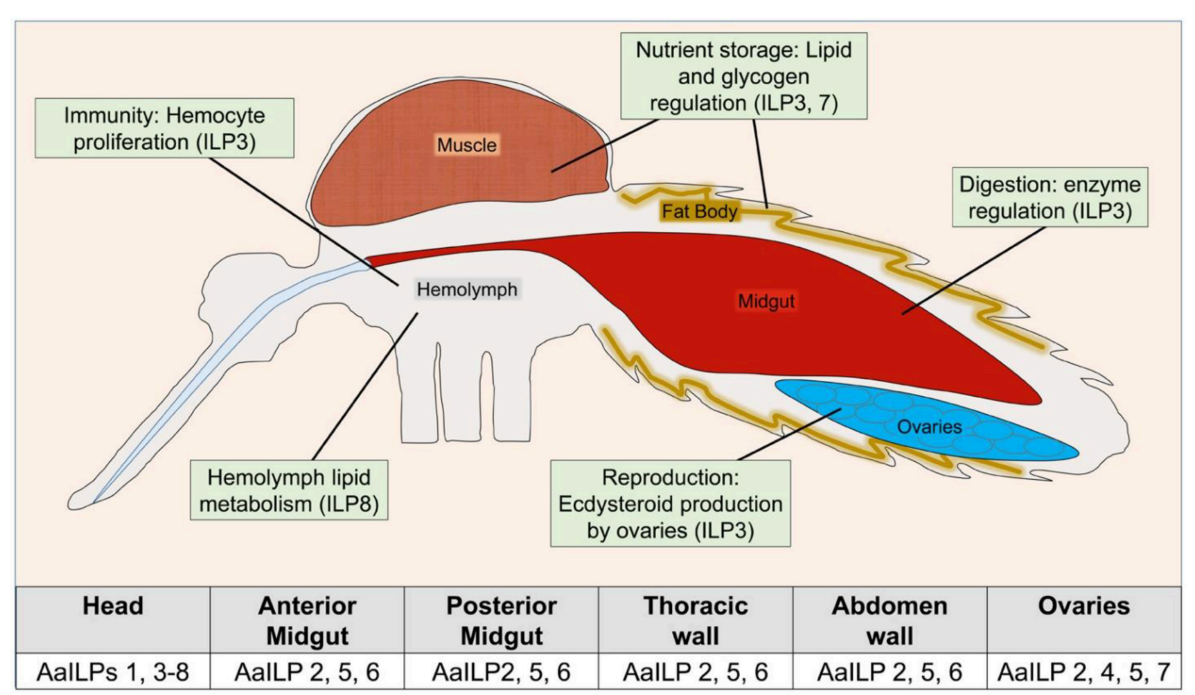

FIGURE 3 | Overview of known ILP functions in Ae. aegypti. Table indicates detection of AalLP expression in adult females, as summarized from (5). For a more detailed description of ILP functions in other species, see Table 2.

\section{Reproduction}

\section{Ecdysteroid and Vitellogenin Production}

The first indication of ILP involvement in insect reproduction was the use of bovine insulin to stimulate ecdysteroid production by in vitro ovaries isolated from unfed female Ae. aegypti (29). Further evidence that this effect was transduced through the insulin signaling complex was provided by using inhibitors or activators of the insulin receptor, $\mathrm{PI} 3 \mathrm{~K}$, and Akt, which altered this response $(6,30,49)$. Bovine insulin in combination with 20-hydroxyecdysone activated transcription of the yolk protein precursor gene and vitellogenin (Vg) in fat body culture. RNAimediated knockdown of the MIR and Akt inhibited insulininduced $\mathrm{Vg}$ gene expression in in vitro fat body culture assays (47). sAaILP3 activated ecdysteroid production in unfed ovaries in vitro $(23,38,50)$. sAaILP4 also stimulated ovaries to produce ecdysteroids in vitro, however, five times higher concentrations of sAaILP4 compared to sAaILP3 were required (39).

Anopheles stephensi sILP3 and sILP4 were both able to stimulate ovaries to produce ecdysteroids in vitro across the genera. Both sAsILPs stimulated ecdysteroid production from unfed ovaries in A. stephensi, A. gambiae, Ae. aegypti, and C. quinquefasciatus (31) suggesting a conserved role of ILPs in the regulation of ecdysteroid productions in mosquitoes. Insulin receptor knockdown in C. quinquefasciatus resulted in low levels ecdysteroids in blood-fed female ovaries (48) further supporting the findings that insulin signaling is required for ecdysteroid production.

\section{Yolk Deposition}

As the blood meal is digested by the female mosquito and nutrients are mobilized, the developing eggs uptake these nutrients as the yolk. Insulin receptor knockdown resulted in a decrease in the amount of yolk deposited in Ae. aegypti ovarioles (23). Injection of sAaILP3 in decapitated, blood-fed females stimulated yolk deposition in $\sim 50 \%$ ovarioles (23), whereas sAaILP3 injection in unfed females stimulated yolk deposition in a $\sim 2 \%$ ovarioles that were later resorbed and never resulted in egg deposition $(41,51)$. RNAi knockdown of PTEN, a negative regulator of insulin receptor substrate, in Ae. aegypti led to an increase in egg production (52) further supporting the role of insulin signaling in reproduction.

CRISPR-Cas9 mutations of AaILP7 and AaILP8 affect ovarian development, but the phenotypes were different. AaILP7 mutant ovaries and their follicles were similar in size to the wild-type at $24 \mathrm{~h}$ post blood meal but were only half the size of those in the control by $72 \mathrm{~h}$. These mosquitoes also had elevated lipid stores at $72 \mathrm{~h}$. In contrast, AaILP8 mutant ovaries were small and melanized by $24 \mathrm{~h}$ post blood meal (40). In $C$. quinquefasciatus females, insulin receptor knockdown and filarial nematode infection resulted in the complete shutdown of egg maturation and deposition (48).

\section{Diapause}

Diapause is characterized by an arrest in ovarian development and the sequestration of large amounts of lipid reserves. The short day lengths program the temperate mosquitoes such as $C$. pipiens to enter a reproductive diapause. Insulin signaling and FOXO (forkhead transcription factor), a downstream molecule in the insulin signaling pathway, are shown to mediate the diapause response (22). In non-diapausing mosquitoes, RNAi knockdown of the insulin receptor led to primary follicles arrested in a stage comparable to diapause. Juvenile hormone application reversed this diapause-like state. When dsRNA directed against FOXO was injected into mosquitoes programmed for diapause, fat storage was dramatically reduced and the mosquito's lifespan was shortened, suggesting that a shutdown of insulin signaling activates the downstream gene FOXO, leading to the diapause phenotype (22). Transcript levels of CpILP1 and 5 were 
significantly lower in diapausing females than in their nondiapausing counterparts (8). Knocking down CpILP1 with RNAi in non-diapausing mosquitoes resulted in a cessation of ovarian development similar to diapausing female mosquitoes, whereas CpILP5 did not alter ovarian development (8).

\section{Lifespan}

The first report of the involvement of insulin signaling in lifespan regulation in invertebrates came from work in Caenorhabditis elegans. In C. elegans, a hypomorphic mutation in the insulin receptor homolog, Daf-2, resulted in a $300 \%$ increase in lifespan (53). In D. melanogaster, hypomorphic insulin receptor expressing flies showed an $85 \%$ increase in lifespan (54). In mosquitoes, overexpression of a myristoylated and active form of A. stephensi and Ae. aegypti Akt in the fat body of transgenic mosquitoes after blood feeding significantly increased adult survivorship relative to non-transgenic sibling controls (55). Similarly, PTEN overexpression also extended mosquito lifespan (56). Therefore, the effect on lifespan in these experiments with mosquitoes seems to be opposite of that seen in C. elegans and D. melanogaster, however, the direct effect of insulin receptor knockdown on lifespan has not yet been studied in mosquitoes. The lack of research is partly due to a lack of easily available genetic tools to make hypomorphic insulin receptor expressing mosquito lines. Most work in mosquitoes is done by RNAi, the effect of which lasts only for 7-10 days. In A. stephensi, high doses of ingested human insulin with blood meal were shown to reduce lifespan (57-59). In contrast, ingested human IGF1 extended lifespan in this species (60).

\section{MOSQUITO IMMUNITY/ MOSQUITO-PATHOGEN INTERACTIONS}

Mosquito hemocytes serve as the most important constitutive defense element against pathogens that enter the hemocoel $(61,62)$ and can produce phagocytic and melanotic immune responses $(63,64)$, effector molecules (65-69), and enhanced defense associated with immune priming (70). Decapitation of A. aegypti mosquitoes after blood feeding inhibited hemocyte proliferation and a single dose of sAaILP3 rescued hemocyte proliferation. Knockdown of the insulin receptor by RNAi inhibited ILP3 rescue activity. This suggests another role of ILPs in hemocyte proliferation, and thus immunity (42).

\section{Malaria Parasite}

The first indication that insulin signaling could play a role in mosquito-pathogen interaction came from a study suggesting human insulin could promote the development of Plasmodium falciparum oocysts in the midguts of $A$. stephensi and $A$. gambiae, although the insulin levels used vastly exceeded those in human blood at the physiological levels (71). P. falciparum glycosylphosphatidylinositols, a parasite factor that mimics insulin in mammals $(72,73)$, was later shown to activate insulin receptor, Akt/PKB (Protein kinase $\mathrm{B}$ ), and the mitogen-activated protein kinase, DSOR 1, in the malaria vector A. stephensi (74).

Human IGFs, within a physiological range and higher levels of human insulin, has been shown to induce nitric oxide (NO) synthesis in mosquito cell culture and in the A. stephensi midgut $(74,75)$. Inducible NO synthesis in A. stephensi limits malaria parasite development through the formation of inflammatory levels of reactive NO that likely induce parasite apoptosis in the mosquito midgut lumen $(57,74,76,77)$. Both radioactive human insulin and IGF1 persisted intact in the midgut up to $30 \mathrm{~h}$ post ingestion and human insulin could activate mosquito insulin receptor by phosphorylation (60).

$P$. falciparum-infected blood meal increased expression of AsILP2, 3, 4, and 5 in the head and midgut of $A$. stephensi (7). Similarly, soluble $P$. falciparum products directly induced AsILP expression in immortalized A. stephensi cells in vitro. Knockdown of AsILP4 by RNAi induced early expression of immune effector genes within $1-6 \mathrm{~h}$ after infection, resulting in significantly reduced parasite abundance prior to invasion of the midgut epithelium. In contrast, knockdown of AsILP3 increased expression of the same genes $24 \mathrm{~h}$ after infection. These data suggest that $P$. falciparum parasites alter the expression of mosquito ILPs to blunt the immune response and facilitate parasite development in the mosquito vector (44). $P$. berghei infection significantly increased AsILP3, 4, and 5 expression. Simultaneous knockdown of AsILP3, 4, and 5 by RNAi reduced $P$. berghei development, yet the difference was not statistically significant (78), whereas insulin receptor knockdown in A. stephensi significantly reduced $P$. berghei development to oocysts (78).

In transgenic A. stephensi, overexpression of Akt in the midgut of heterozygous mosquitoes resulted in $60-99 \%$ reduction in the numbers of mosquitoes infected with $P$. falciparum, and parasite infection was completely blocked in homozygous transgenic mosquitoes (79). In addition, a single nucleotide polymorphism (SNP) in the AgILP3 gene (Ins34) was reported in field-collected A. gambiae mosquitoes from Mali (45). This synonymous SNP in Ins34 in AgILP3 precursor gene resulted in a change from GGC to GGT at nucleotide position 462 . The CC genotype at the Ins34 locus in $\mathrm{M}$ form mosquitoes was more common in samples that were not infected with $P$. falciparum suggesting a role of this pathway in malaria parasite infection.

\section{Filarial Parasites}

Insulin receptor knockdown in C. quinquefasciatus, the major vector of Wuchereria bancrofti in India, completely blocked the development of filarial nematode parasites to the infective third instar larval stage (48). This is the only study on the role of mosquito insulin signaling in nematode development and the data suggest a conserved role of insulin signaling in parasite development within mosquito vectors.

\section{CONCLUSIONS}

Insulin-like peptides are pleiotropic peptide hormones, and owing to this, the structural and functional characterization of ILPs has long been a major interest for insect endocrinologists. The functions of insect ILPs, in general, is in a discovery phase compared to the state of knowledge for insulins and related peptides in vertebrates. A primary action of insulin in mammals is to reduce circulating glucose through increased glycogen and 
triglyceride synthesis. This action is conserved in mosquitoes and has been supported by the work in Ae. aegypti. Over the past several years, a succession of studies has suggested a central role of ILPs/insulin signaling in regulating growth, development, reproduction, diapause, aging, and pathogen development in mosquitoes. Yet, only a few studies have used genetic tools to dissect out the functions of individual ILPs. Unraveling the individual functions and functional redundancy of ILPs will provide new understanding of this complex pathway.

\section{FUTURE GOALS}

It is clear from the review of literature that there are many unanswered questions regarding the roles of insulin signaling in mosquitoes. Only a few mosquito ILPs have been functionally characterized, mostly because of the challenges in peptide purification and synthesis, efficient transcript knockdown, and potentially overlapping functions. So far only one endogenous mosquito ILP has been purified and characterized, limiting our understanding of the post-translational processing of these molecules, and there is no data on ILPs that may fold similarly to IGF-I, without cleavage of the C-peptide (e.g., AaILP6). In addition, cell-based expression systems have so far been unable to produce biologically active mosquito ILPs, and chemical ILP synthesis has been difficult due to complex formation of disulfide bridges between multiple Cys residues and proper cleavage of the C-peptide required for folding. Both Ae. aegypti and D. melanogaster research has benefitted from a handful synthetic ILPs, albeit at a high synthesis cost, and availability of more synthetic ILPs would inform ligand-receptor interactions as well as ILP functions. Also, a standard HPLC or GC-MS protocol for measuring ILPs titers would also help improve our knowledge of ILP functions, either as circulating hormones or as a neurotransmitters.

\section{REFERENCES}

1. De Meyts P, Gauguin L, Svendsen AM, Sarhan M, Knudsen L, Nøhr J, et al. Structural basis of allosteric ligand-receptor interactions in the insulinrelaxin peptide family: implications for other receptor tyrosine kinases and G-protein-coupled receptors. Ann NY Acad Sci. (2009) 1160:45-53. doi: 10.1111/j.1749-6632.2009.03837.x

2. Colombani J, Andersen DS, Léopol P. Secreted peptide dilp8 coordinates Drosophila tissue growth with developmental timing. Science. (2012) 336:5825. doi: 10.1126/science.1216689

3. Garelli A, Gontijo AM, Miguela V, Caparros E, Dominguez M. Imaginal discs secrete insulin-like peptide 8 to mediate plasticity of growth and maturation. Science. (2012) 336:579-82. doi: 10.1126/science.1216735

4. Nässel DR. Insulin-producing cells and their regulation in physiology and behavior of Drosophila. Can J Zool. (2012) 50:476-88. doi: 10.1139/z20 12-009

5. Riehle MA, Fan Y, Cao C, Brown MR. Molecular characterization of insulin-like peptides in the yellow fever mosquito, Aedes aegypti: expression, cellular localization, and phylogeny. Peptides. (2006) 27:2547-60. doi: 10.1016/j.peptides.2006.07.016

6. Riehle MA, Brown MR. Insulin receptor expression during development and a reproductive cycle in the ovary of the mosquito Aedes aegypti. Cell Tissue Res. (2002) 308:409-20. doi: 10.1007/s00441-002-0561-8
Recent advances in gene editing technologies now allow explorations of ILP functions outside of model organisms. Most notably, CRISPR-Cas9 knock-ins or knock-outs will facilitate acquisition of new knowledge on how ILPs control physiologies such as nutrient storage, lifespan, development, fecundity, host seeking (appetite), regulation of proteases, and immune response. Genetic knockouts allow for persistent, lifelong knockouts or overexpression mutants which could not previously be achieved through RNAi or injection of synthetic peptides. Additionally, the use of an epitope tag such as HA coupled with single guide RNA (sgRNA) in a donor construct along with a fluorescent marker could be a tool for simultaneously tracking ILP expression locations and patterns, even when the hormone itself is knocked out. With the improvement of gene editing in mosquitoes, it will be possible to understand the functions of ILPs in more mosquito species in addition to Ae. aegypti, An. gambiae, and An. stephensi in order to understand if ILP functions are conserved or change in different mosquito taxa.

An area of research that is clearly open is the role of insulin signaling in mosquito-pathogen interactions. Therefore, research should focus on understanding the diverse functions of ILPs in mosquitoes including mosquito-pathogen interactions. Insulin mimetics that bind to the insulin receptor and block the downstream processes might be a new avenue to explore for mosquito and mosquito-borne disease control.

\section{AUTHOR CONTRIBUTIONS}

AS, AN, and MG-N wrote the draft manuscript. AN and MG-N wrote the final manuscript.

\section{ACKNOWLEDGMENTS}

We are thankful to Manoj Mathew for mosquito rearing.

7. Marquez AG, Pietri JE, Smithers HM, Nuss A, Antonova Y, Drexler AL, et al. Insulin-like peptides in the mosquito Anopheles stephensi: identification and expression in response to diet and infection with Plasmodium falciparum. Gen Comp Endocrinol. (2011) 173:303-12. doi: 10.1016/j.ygcen.2011.06.005

8. Sim C, Denlinger DL. A shut-down in the expression of an insulinlike peptide, ILP-1, halts ovarian maturation during the overwintering diapause of the mosquito Culex pipiens. Insect Mol Biol. (2009) 18:325-32. doi: 10.1111/j.1365-2583.2009.00872.x

9. Riehle MA, Garczynski SF, Crim JW, Hill CA, Brown MR. Neuropeptides and peptide hormones in Anopheles gambiae. Science. (2002) 298:172-5. doi: 10.1126/science.1076827

10. White MF. The IRS-signaling system: a network of docking proteins that mediate insulin action. Mol Cell Biochem. (1998) 182:3-11. doi: 10.1023/A:1006806722619

11. Yenush L, Fernandez R, Myers MG Jr, Grammer TC, Sun XJ, Blenis J, et al. The Drosophila insulin receptor activates multiple signaling pathways but requires insulin receptor substrate proteins for DNA synthesis. Mol Cell Biol. (1996) 16:2509-17. doi: 10.1128/MCB.16.5.2509

12. Blenis J. Signal transduction via the MAP kinases: proceed at your own RSK. Proc Natl Acad Sci USA. (1993) 90:5889-92. doi: 10.1073/pnas.90.13.5889

13. Shepherd PR, Withers DJ, Siddle K. Phosphoinositide 3-kinase: the key switch mechanism in insulin signaling. Biochem J. (1998) 333:471-90. doi: 10.1042/bj3330471 
14. Alessi DR, Cohen P. Mechanism of activation and function of protein kinase B. Curr Opin Genet Dev. (1998) 8:55-62. doi: 10.1016/S0959-437X(98) 80062-2

15. Oldham S, Montagne J, Radimerski T, Thomas G, Hafen E. Genetic and biochemical characterization of dTOR, the Drosophila homolog of the target of rapamycin. Genes Dev. (2000) 14:2689-94. doi: 10.1101/gad.845700

16. Huang J, Manning BD. A complex interplay between Akt, TSC2 and the two mTOR complexes. Biochem Soc Trans. (2009) 37:217-22. doi: 10.1042/BST0370217

17. Lin K, Dorman JB, Rodan A, Kenyon C. daf-16: an HNF-3/forkhead family member that can function to double the life-span of Caenorhabditis elegans. Science. (1997) 278:1319-22. doi: 10.1126/science.278.5341.1319

18. Ogg S, Paradis S, Gottlieb S, Patterson GI, Lee L, Tissenbaum HA, Ruvkun G. The forkhead transcription factor DAF-16 transduces insulinlike metabolic and longevity signals in C. elegans. Nature. (1997) 389:994-9. doi: 10.1038/40194

19. Brunet A, Bonni A, Zigmond MJ, Lin MZ, Juo P, Hu LS, et al. Akt promotes cell survival by phosphorylating and inhibiting a forkhead transcription factor. Cell. (1999) 96:857-68. doi: 10.1016/S0092-8674(00)80595-4

20. Kops GJPL, De Ruiter ND, De Vries-Smits AMM, Powell DR, Bos JL, Burgering BMT. Direct control of the forkhead transcription factor AFX by protein kinase B. Nature. (1999) 398:630-4. doi: 10.1038/19328

21. Kramer JM, Slade JD, Staveley BE. foxo is required for resistance to amino acid starvation in Drosophila. Genome. (2008) 51:668-72. doi: 10.1139/G08-047

22. Sim C, Denlinger DL. Insulin signaling and FOXO regulate the overwintering diapause of the mosquito Culex pipiens. Proc Natl Acad Sci USA. (2008) 105:6777-81. doi: 10.1073/pnas.0802067105

23. Gulia-Nuss M, Robertson AE, Brown MR, Strand MR. Insulin-like peptides and the target of rapamycin pathway coordinately regulate blood digestion and egg maturation in the mosquito Aedes aegypti. PLoS ONE. (2011) 6:e20401. doi: 10.1371/journal.pone.0020401

24. Sim C, Denlinger DL. Juvenile hormone III suppresses forkhead of a transcription factor in the fat body and reduces fat accumulation in the diapausing mosquito, Culex pipiens. Insect Mol Biol. (2013) 22:1-11. doi: 10.1111/j.1365-2583.2012.01166.x

25. Sim C, Kang DS, Kim S, Bai X, Denlinger DL. Identification of FOXO targets that generate diverse features of the diapause phenotype in the mosquito Culex pipiens. Proc Natl Acad Sci USA. (2015) 112:3811-6. doi: 10.1073/pnas.1502751112

26. Perez-Hedo M, Rivera-Perez C, Noriega FG. Starvation increases insulin sensitivity and reduces juvenile hormone synthesis in mosquitoes. PLOS ONE. (2014) 9:e0086183. doi: 10.1371/journal.pone.0086183

27. Hansen IA, Sieglaff DH, Munro JB, Shiao SH, Cruz J, Lee IW, et al. Forkhead transcription factors regulate mosquito reproduction. Insect Biochem Mol Biol. (2014) 37:985-97. doi: 10.1016/j.ibmb.2007.05.008

28. Roy SG, Raikhel AS. Nutritional and hormonal regulation of the TOR effector $4 \mathrm{E}-$ binding protein (4E-BP) in the mosquito Aedes aegypti. FASEB J. (2012) 26:1334-42. doi: 10.1096/fj.11-189969

29. Graf R, Neuenschwander S, Brown MR, Ackermann U. Insulin-mediated secretion of ecdysteroids from mosquito ovaries and molecular cloning of the insulin receptor homolog from ovaries of blood-fed Aedes aegypti. Insect Mol Biol. (1997) 6:151-63. doi: 10.1111/j.1365-2583.1997.tb0 0083.x

30. Riehle MA, Brown MR. Insulin stimulates ecdysteroid production through a conserved signaling cascade in the mosquito Aedes aegypti. Insect Biochem Mol Biol. (1999) 29:855-60. doi: 10.1016/S0965-1748(99)0 0084-3

31. Nuss AB, Brown MR. Isolation of an insulin-like peptide from the Asian malaria mosquito, Anopheles stephensi, that acts as a steroidogenic gonadotropin across diverse mosquito taxa. Gen Comp Endocrinol. (2018) 258:140-8. doi: 10.1016/j.ygcen.2017.05.007

32. Combet C, Blanchet C, Geourjon C, Deléage G. NPS@: network protein sequence analysis. Trends Biochem Sci. (2000) 25:147-50. doi: 10.1016/S0968-0004(99)01540-6

33. Robert X, Gouet P. Deciphering key features in protein structures with the new ENDscript server. Nucleic Acids Res. (2014) 42:W320-4. doi: 10.1093/nar/gku316
34. Tamura K, Stecher G, Peterson D, Filipski A, Kumar S. MEGA6: molecular evolutionary genetics analysis version 6.0. Mol Biol Evol. (2013) 30:2725-9. doi: 10.1093/molbev/mst197

35. Antonova Y, Arik AJ, Moore W, Riehle MA, Brown MR. Insulinlike peptides: structure, signaling, and function. In: Gilbert LI, editor. Insect Endocrinology. 1st ed. New York, NY: Elsevier (2012). p. 63-92. doi: 10.1016/B978-0-12-384749-2.10002-0

36. Strand MR, Brown MR, Vogel KJ. Mosquito peptide hormones: diversity, production, and function. Adv Insect Physiol. (2016) 51:145-88. doi: 10.1016/bs.aiip.2016.05.003

37. Nässel DR, Broeck JV. Insulin/IGF signaling in Drosophila and other insects: factors that regulate production, release and post-release action of the insulin-like peptides. Cell Mol Life Sci. (2016) 73:271-90. doi: 10.1007/s00018-015-2063-3

38. Brown MR, Clark KD, Gulia M, Zhao Z, Garczynski SF, Crim JW, et al. An insulin-like peptide regulates egg maturation and metabolism in the mosquito Aedes aegypti. Proc Natl Acad Sci USA. (2008) 105:5716-21. doi: 10.1073/pnas.0800478105

39. Wen Z, Gulia M, Clark KD, Dhara A, Crim JW, Strand MR, et al. Two insulin-like peptide family members from the mosquito Aedes aegypti exhibit differential biological and receptor binding activities. Mol Cell Endocrinol. (2010) 328:47-55. doi: 10.1016/j.mce.2010.07.003

40. Ling L, Kokoza VA, Zhang C, Aksoy E, Raikhel AS. MicroRNA-277 targets insulin-like peptides 7 and 8 to control lipid metabolism and reproduction in Aedes aegypti mosquitoes. Proc Natl Acad Sci USA. (2017) 114:E8017-24. doi: $10.1073 /$ pnas.1710970114

41. Gulia-Nuss M, Elliot A, Brown MR, Strand MR. Multiple factors contribute to anautogenous reproduction by the mosquito Aedes aegypti. J Insect Physiol. (2015) 82:8-16. doi: 10.1016/j.jinsphys.2015.08.001

42. Castillo J, Brown MR, Strand MR. Blood feeding and insulin-like peptide 3 stimulate proliferation of hemocytes in the mosquito Aedes aegypti. PLoS Pathogens. (2011) 7:e1002274. doi: 10.1371/journal.ppat.1002274

43. Pooriirouby R, Sharma A, Beard J, Reyes J, Nuss A, Gulia-Nuss M. Nutritional quality during development alters insulin-like peptides expression and physiology of the adult yellow fever mosquito, Aedes aegypti. Insects. (2018) 9:110 doi: 10.3390/insects9030110

44. Pietri JE, Pietri EJ, Potts R, Riehle MA, Luckhart S. Plasmodium falciparum suppresses the host immune response by inducing the synthesis of insulinlike peptides (ILPs) in the mosquito Anopheles stephensi. Dev Comp Immunol. (2015) 53:134-44. doi: 10.1016/j.dci.2015.06.012

45. Horton AA, Lee Y, Coulibaly CA, Rashbrook VK, Cornel AJ, Lanzaro GC, et al. Identification of three single nucleotide polymorphisms in Anopheles gambiae immune signaling genes that are associated with natural Plasmodium falciparum infection. Malaria J. (2010) 9:160. doi: 10.1186/1475-28759-160

46. Arsic D, Guerin PM. Nutrient content of diet affects the signaling activity of the insulin/target of rapamycin/p70 S6 kinase pathway in the African malaria mosquito Anopheles gambiae. J Insect Physiol. (2008) 54:1226-35. doi: 10.1016/j.jinsphys.2008.06.003

47. Roy SG, Hansen IA, Raikhel AS. Effect of insulin and 20-hydroxyecdysone in the fat body of the yellow fever mosquito, Aedes aegypti. Insect Biochem Mol Biol. (2007) 37:1317-26. doi: 10.1016/j.ibmb.2007.08.004

48. Nuss AB, Brown MR, Murty US, Gulia-Nuss M. Insulin receptor knockdown blocks filarial parasite development and alters egg production in the southern house mosquito, Culex quinquefasciatus. PLoS Neglect Trop Dis. (2018) 12:e006413. doi: 10.1371/journal.pntd.0006413

49. Riehle MA, Brown MR. Molecular analysis of the serine/threonine kinase Akt and its expression in the mosquito Aedes aegypti. Insect Mol Biol. (2003) 12:225-32. doi: 10.1046/j.1365-2583.2003.00405.x

50. Dhara A, Eum JH, Robertson A, Gulia-Nuss M, Vogel KJ, Clark KD, et al. Ovary ecdysteroidogenic hormone functions independently of the insulin receptor in the yellow fever mosquito, Aedes aegypti. Insect Biochem Mol Biol. (2013) 43:1100-8. doi: 10.1016/j.ibmb.2013.09.004

51. Gulia-Nuss M, Eum J-H, Strand MR, Brown MR. Ovary ecdysteroidogenic hormone activates egg maturation in the mosquito Georgecraigius atropalpus after adult eclosion or a blood meal. J Exp Biol. (2012) 215:3758-67. doi: $10.1242 /$ jeb.074617 
52. Arik AJ, Rasgon JL, Quicke KM, Riehle MA. Manipulating insulin signaling to enhance mosquito reproduction. BMC Physiol. (2009) 9:15. doi: $10.1186 / 1472-6793-9-15$

53. Kenyon C, Chang J, Gensch E, Rudner A, Tabtiang R. A C. elegans mutant that lives twice as long as wild-type. Nature. (1993) 366:461-4. doi: 10.1038/366461a0

54. Tatar M, Kopelman A, Epstein D, Tu MP, Yin CM, Garofalo RS. A mutant Drosophila insulin receptor homolog that extends life-span and impairs neuroendocrine function. Science. (2001) 292:107-10. doi: 10.1126/science.1057987

55. Arik AJ, Hun LV, Quicke K, Piatt M, Ziegler R, Scaraffia PY, et al. Increased Akt signaling in the mosquito fat body increases adult survivorship. FASEB J. (2015) 29:1404-13. doi: 10.1096/fj.14-261479

56. Hauck ES, Antonova-Koch Y, Drexler A, Pietri J, Pakpour N, Liu D, et al. Overexpression of phosphatase and tensin homolog improves fitness and decreases Plasmodium falciparum development in Anopheles stephensi. Microbes Infect. (2013) 15:775-87. doi: 10.1016/j.micinf.2013.05.006

57. Kang M-A, Mott TM, Tapley EC, Lewis EE, Luckhart S. Insulin regulates aging and oxidative stress in Anopheles stephensi. J Exp Biol. (2008) 211:741-8. doi: 10.1242/jeb.012955

58. Surachetpong W, Singh N, Cheung KW, Luckhart S. MAPK ERK signaling regulates the TGF-b $\beta 1$-dependent mosquito response to Plasmodium falciparum. PLoS Pathogens. (2009) 5:e1000366. doi: 10.1371/journal.ppat.1000366

59. Pakpour N, Corby-Harris V, Green GP, Smithers HM, Cheung KW, Riehle $\mathrm{MA}$, et al. Ingested human insulin inhibits the mosquito NF-кB-dependent immune response to Plasmodium falciparum. Infect Immun. (2012) 80:21419. doi: 10.1128/IAI.00024-12

60. Drexler A, Nuss A, Hauck E, Glennon E, Cheung K, Brown M, et al. Human IGF1 extends lifespan and enhances resistance to Plasmodium falciparum infection in the malaria vector Anopheles stephensi. J Exp Biol. (2013) 216:20817. doi: $10.1242 /$ jeb.078873

61. Strand MR. The insect cellular immune response. Insect Sci. (2008) 15:1-14. doi: 10.1111/j.1744-7917.2008.00183.x

62. Hillyer JF. Mosquito immunity. Adv Exp Med Biol. (2010) 708:218-38. doi: 10.1007/978-1-4419-8059-5-12

63. Hillyer JF, Schmidt SL, Christensen BM. Rapid phagocytosis and melanization of bacteria and plasmodium. J Parasitol. (2003) 89:62-9. doi: 10.1645/00223395(2003)089[0062:RPAMOB]2.0.CO;2

64. Hillyer JF, Schmidt SL, Fuchs JF, Boyle JP, Christensen BM. Age-associated mortality in immune-challenged mosquitoes (Aedes aegypti) correlates with a decrease in haemocyte numbers. Cell Microbiol. (2005) 7:39-51. doi: 10.1111/j.1462-5822.2004.00430.x

65. Baton LA, Robertson A, Warr E, Strand MR, Dimopoulos G. Genome-wide transcriptomic profiling of Anopheles gambiae hemocytes reveals pathogenspecific signatures upon bacterial challenge and Plasmodium berghei infection. BMC Genomics. (2009) 10:257. doi: 10.1186/1471-2164-10-257

66. Pinto SB, Lombardo F, Koutsos AC, Waterhouse RM, McKay K, An C, et al. Discovery of Plasmodium modulators by genome-wide analysis of circulating hemocytes in Anopheles gambiae. Proc Natl Acad Sci USA. (2009) 106:212705. doi: 10.1073/pnas.0909463106

67. Blandin SA, Marois E, Levashina EA. Antimalarial responses in Anopheles gambiae: from a complement-like protein to a complement-like pathway. Cell Host Microbe. (2008) 3:364-74. doi: 10.1016/j.chom.2008.05.007
68. Bartholomay LC, Mayhew GF, Fuchs JF, Rocheleau TA, Erickson SM, Aliota MT. Profiling infection responses in the haemocytes of the mosquito, Aedes aegypti. Insect Mol Biol. (2007) 16:761-76. doi: 10.1111/j.1365-2583.2007.00773.x

69. Abraham EG, Pinto SB, Ghosh A, Vanlandingham DL, Budd A, Higgs S, et al. An immune-responsive serpin, SRPN6, mediates mosquito defense against malaria parasites. Proc Natl Acad Sci USA. (2005) 102:16327-32. doi: 10.1073/pnas.0508335102

70. Rodrigues J, Brayner FA, Alves LC, Dixit R, Barillas-Mury C. Hemocyte differentiation mediates innate immune memory in Anopheles gambiae mosquitoes. Science. (2010) 329:1353-5. doi: 10.1126/science.1190689

71. Beier MS, Pumpuni CB, Beier JC, Davis JR. Effects of para-aminobenzoic acid, insulin, and gentamicin on Plasmodium falciparum development in Anopheline mosquitoes (Diptera: Culicidae). J Med Entomol. (1994) 31:561-5. doi: 10.1093/jmedent/31.4.561

72. Schofield L, Hackett F. Signal transduction in host cells by a glycosylphosphatidylinositol toxin of malaria parasites. J Exp Med. (1993) 177:145-53. doi: 10.1084/jem.177.1.145

73. Caro HN, Sheikh NA, Taverne J, Playfair JHL, Rademacher TW. Structural similarities among malaria toxins, insulin second messengers, and bacterial endotoxin. Infect Immun. (1996) 64:3438-41.

74. Lim J, Gowda DC, Krishnegowda G, Luckhart S. Induction of nitric oxide synthase in Anopheles stephensi by Plasmodium falciparum: mechanism of signaling and the role of parasite glycosylphosphatidylinositols. Infect Immun. (2005) 73:2778-89. doi: 10.1128/IAI.73.5.2778-2789.2005

75. Drexler AL, Pietri JE, Pakpour N, Hauck E, Wang B, Glennon EKK, et al. Human IGF1 regulates midgut oxidative stress and epithelial homeostasis to balance lifespan and Plasmodium falciparum resistance in Anopheles stephensi. PLoS Pathogens. (2014) 10:e1004231. doi: 10.1371/journal.ppat.1004231

76. Luckhart S, Vodovotz Y, Cui L, Rosenberg R. The mosquito Anopheles stephensi limits malaria parasite development with inducible synthesis of nitric oxide. Proc Natl Acad Sci USA. (1998) 95:5700-5.

77. Peterson TML, Gow AJ, Luckhart S. Nitric oxide metabolites induced in Anopheles stephensi control malaria parasite infection. Free Rad Biol Med. (2007) 42:132-42. doi: 10.1016/j.freeradbiomed.2006.10.037

78. Zhao YO, Kurscheid S, Zhang Y, Liu L, Zhang L, Loeliger K, et al. Enhanced survival of Plasmodium-infected mosquitoes during starvation. PLoS ONE. (2012) 7:e40556. doi: 10.1371/journal.pone.0040556

79. Corby-Harris V, Drexler A, de Jong LW, Antonova Y, Pakpour N, Ziegler $\mathrm{R}$, et al. Activation of Akt signaling reduces the prevalence and intensity of malaria parasite infection and lifespan in Anopheles stephensi mosquitoes. PLoS Pathogens. (2010) 6:1-10. doi: 10.1371/journal.ppat.1001003

Conflict of Interest Statement: The authors declare that the research was conducted in the absence of any commercial or financial relationships that could be construed as a potential conflict of interest.

Copyright (c) 2019 Sharma, Nuss and Gulia-Nuss. This is an open-access article distributed under the terms of the Creative Commons Attribution License (CC BY). The use, distribution or reproduction in other forums is permitted, provided the original author(s) and the copyright owner(s) are credited and that the original publication in this journal is cited, in accordance with accepted academic practice. No use, distribution or reproduction is permitted which does not comply with these terms. 